\title{
High-resolution two-dimensional map of the solar time anisotropy obtained by the GRAPES-3 large-area muon telescope
}

\author{
H. Kojima, ${ }^{1, a, k}$ S. Ahmad, ${ }^{1, d}$ M. Chakraborty, ${ }^{1, b}$ A. Chandra, ${ }^{1, d}$ S. R. Dugad, ${ }^{1, b}$ \\ S. K. Gupta, ${ }^{1, b, k}$ B. Hariharan, ${ }^{1, b}$ Y. Hayashi, ${ }^{1, c}$ P. Jagadeesan, ${ }^{1, b}$ A. Jain, ${ }^{1, b}$ P. Jain, ${ }^{1, e}$ \\ V. B. Jhansi, ${ }^{1, b}$ S. Kawakami, ${ }^{1, c}$ T. Koi, ${ }^{1, a}$ S. Mahapatra, ${ }^{1, f}$ T. Matsuyama, ${ }^{1, c}$ \\ P. K. Mohanty, ${ }^{1, b}$ I. Morishita, ${ }^{1, h}$ S. D. Morris, ${ }^{1, b}$ T. Nakamura, ${ }^{1, j}$ P. K. Nayak, ${ }^{1, b}$ \\ T. Nonaka, ${ }^{1, g}$ S. Ogio, ${ }^{1, c}$ A. Oshima, ${ }^{1, a, k}$ D. Pattanaik, ${ }^{1, b}$ P. S. Rakshe, ${ }^{1, b}$ \\ K. Ramesh, ${ }^{1, b}$ B. S. Rao, ${ }^{1, b}$ L. V. Reddy, ${ }^{1, b}$ S. Shibata, ${ }^{1, a, k}$ H. Takamaru, ${ }^{1, a, k}$ \\ K. Tanaka, ${ }^{1, i}$ F. Varsi, ${ }^{1, e}$ K. Yamazaki ${ }^{1, a, *}$ and M. Zuberi ${ }^{1, b}$ \\ ${ }^{a}$ College of Engineering, Chubu University, Kasugai, Aichi 487-8501, Japan \\ ${ }^{b}$ Tata Institute of Fundamental Research, Mumbai 400005, India \\ ${ }^{c}$ Graduate School of Science, Osaka City University, Osaka 558-8585, Japan \\ ${ }^{d}$ Aligarh Muslim University, Aligarh 202002, India \\ ${ }^{e}$ Indian Institute of Technology Kanpur, Kanpur 208016, India \\ ${ }^{f}$ Utkal University, Bhubaneshwar 751004, India \\ ${ }^{g}$ Institute for Cosmic Ray Research, Tokyo University, Kashiwa, Chiba 277-8582, Japan \\ ${ }^{h}$ Asahi University, Mizuho, Gifu 501-0296, Japan \\ ${ }^{i}$ Graduate School of Information Sciences, Hiroshima City University, Hiroshima 731-3194, Japan \\ ${ }^{j}$ Kochi University, Kochi, Kochi 780-8072, Japan \\ ${ }^{k}$ Astronomical Observatory, Chubu University, Kasugai, Aichi 487-8501, Japan \\ E-mail: hkojima@isc.chubu.ac.jp
}

In the analysis of the anisotropy of the galactic cosmic rays caused by the solar modulation below $1 \mathrm{TeV}$, time series graphs with time on the horizontal axis and cosmic ray intensity as the vertical axis had been widely adopted and other style of the graphs called harmonic vector diagrams had also been used. These methods are the time series analyses, and because of the horizontal axis (time), the graph is often misinterpreted as a "temporal variation," even though it must be regarded as an intrinsically "spatial distribution."

This paper presents a high-resolution two-dimensional map of the solar time anisotropy using the GRAPES-3 large-area muon telescope at Ooty, south India, which has an excellent capability of observing the muon intensity in 169 directions within a $50^{\circ}$ zenith angle.

$37^{\text {th }}$ International Cosmic Ray Conference (ICRC 2021)

July 12 th - 23rd, 2021

Online - Berlin, Germany

${ }^{1}$ For the GRAPES-3 collaboration.

*Presenter 


\section{Introduction}

Galactic cosmic rays (GCR) enter the heliosphere and propagate to the Earth through the solar magnetic field frozen in the solar wind (so-called interplanetary magnetic field, IMF). So the flow of GCR reflects the structure (strength, direction, polarity) of IMF controlled by solar activities. Therefore, knowing the directional distribution of GCR is a clue to investigate the aspect of the magnetic field in interplanetary space on a scale corresponding to the rigidity of the GCR, and this is a key to know the so-called "space weather."

In traditional analysis, especially for GCR below $1 \mathrm{TeV}$ (e.g. [1-5]), a time series graph with time on the horizontal axis and cosmic ray intensity on the vertical axis is used as shown in Figure 1 or the harmonic vector diagrams as shown in Figure 2, even in one of recent very important works on the solar diurnal variations [6].
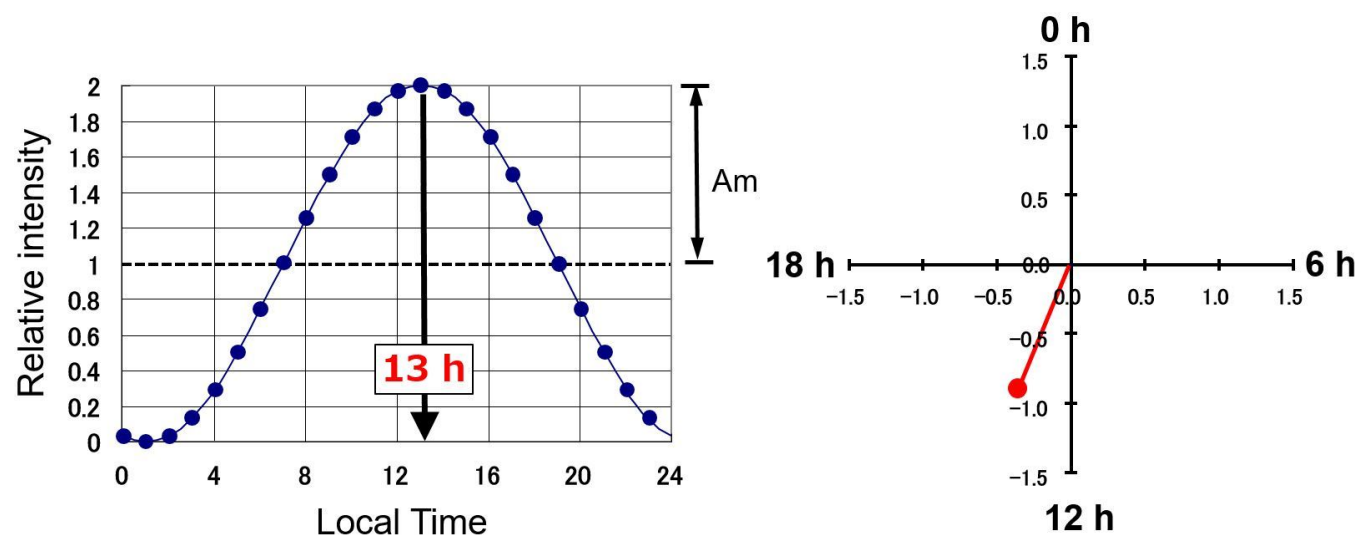

Figure 1: An example of the time series graphs of Cosmic Ray intensity.

Figure 2: An example of the harmonic diagrams.

A method mentioned above is the so-called time series analysis. Because of the horizontal axis (time), the graph is often misinterpreted as a "temporal variation," even though it must be regarded as an intrinsically "spatial distribution (as shown in Figure 3)."

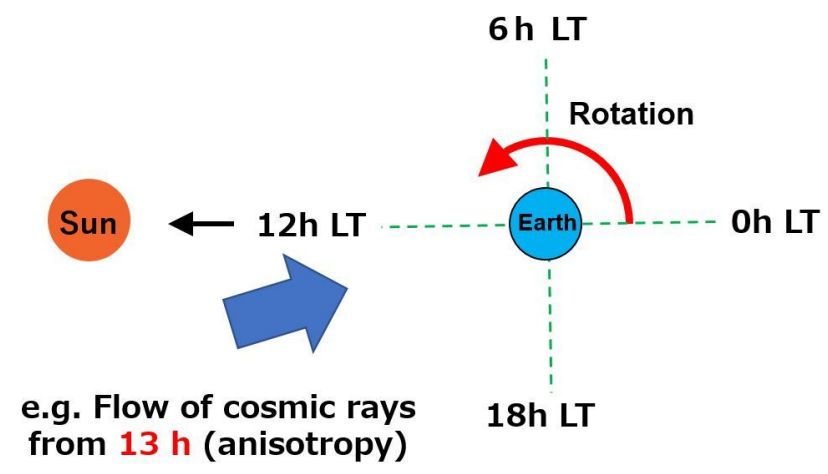

Figure 3: Flow of GCR from a direction of $13 \mathrm{~h}\left(15^{\circ}\right.$ east from the Sun $)$ fixed in the geocentric solar coordinate system. An interpretation in the case of Figure 1 or Figure 2. 
In the case of the study of solar modulation, we use the solar time as the basis of the analysis. Namely the Earth's rotation in one solar day gives a single scan around the Earth. According to this view, if we obtain the time series graphs like Figure 1 for more than several days, we interpret the phenomena as the flow of GCR near the Earth might be coming from a direction of $13 \mathrm{~h}\left(15^{\circ}\right.$ east from the Sun) fixed in the geocentric solar coordinate system as shown in Figure 3, that stands stationary in the corresponding duration.

In the above example, we assumed that we had observed the intensity of GCR with a single detector placed somewhere on the surface of the Earth. Then we only obtained the intensity variation of GCR on a trace of the sky with some fixed declination. If we have more than one detector, then we will get much information about the intensity of GCR on the sky.

From this viewpoint, we have an advantage that we already have been operating the large area and high-resolution muon telescope at Ooty, south India, which can observe the muon intensity in 169 directions as described in the next section. Utilizing this muon telescope, we have studied the transient phenomena of GCR intensity variation during Forbush Decrease [7-10] and the intensity of muons affected by thundercloud [11]. Between them, three works [7, 8, 11] have been performed by the two-dimensional (2D) visualization method. This paper proposes to apply this $2 \mathrm{D}$ visualization method to much longer time scale phenomena.

In the third section, we will present a high-resolution 2D map that will show the steady-state solar time anisotropy of GCR observed during 14 years from 2004 to 2017 by the large area and high-resolution GRAPES-3 muon telescope.

\section{The GRAPES-3 large-area muon telescope}

The GRAPES-3 (Gamma Ray Astronomy at PeV EnergieS phase-3) experiment was set up at Ooty $\left(11.4^{\circ} \mathrm{N}\right.$ latitude, $76.7^{\circ} \mathrm{E}$ longitude, and 2,200 $\mathrm{m}$ altitude), south India. The GRAPES-3 experiment consists of two major components. The first component is an extensive air shower (EAS) array of compact configuration with an inter-detector separation of $8 \mathrm{~m}$ between the adjacent plastic scintillator detectors, each $1 \mathrm{~m}^{2}$ in the area [12]. The second major component of the GRAPES-3 experiment is a large area tracking muon telescope that has been used to measure the muon content of the EAS [13]. This information is critically important in determining the composition of the primary cosmic rays [14-17]. This unique instrument has also been used to measure the variation of GCR intensity due to the solar activity $[1-5,7-10,18,19]$. The muon telescope is capable of providing a high statistics, directional measurement of the muons.

The GRAPES-3 muon telescope consists of a total of 16 individual modules. The basic element of the muon telescope is the proportional counter (PRC) fabricated from a rugged 600 $\mathrm{cm}$ long seamless steel pipe with a $2.3 \mathrm{~mm}$ thick wall and a square cross-section of $10 \times 10 \mathrm{~cm}^{2}$. The concrete blocks have been arranged in the shape of an inverted pyramid to achieve an energy threshold $1 \sec (\theta) \mathrm{GeV}$ for muons incident on the detector with a zenith angle $\theta$.

A module of sensitive area $35 \mathrm{~m}^{2}$ consists of 232 PRCs arranged in four layers of 58 PRCs each. The four layers of PRCs, namely, layers-0, 1, 2, and 3 in mutually orthogonal directions, are interleaved with absorber concrete blocks of $15 \mathrm{~cm}$ thick, as shown schematically in Figure 4. So the vertical separation of the two PRC layers in the same projection plane is $\sim 50 \mathrm{~cm}$, allowing 
reconstruction of each muon track and determination of the arrival direction with an accuracy of $\leq 6^{\circ}$ in each projected plane.

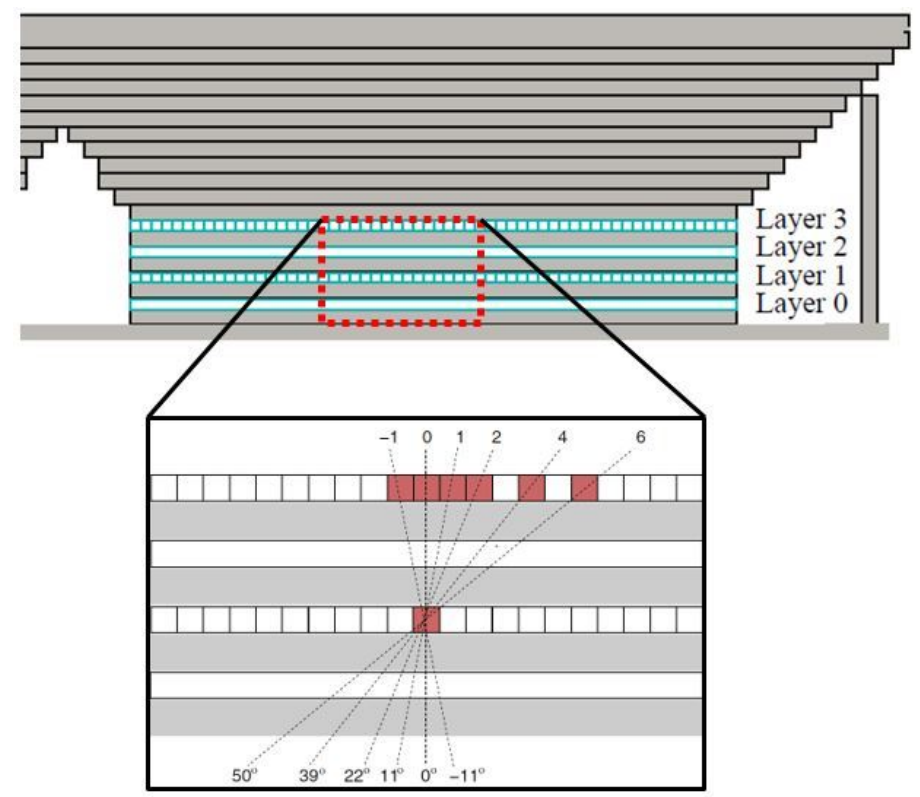

Figure 4: A cross section of the muon telescope and muon projection angles $\left(0^{\circ}, 11^{\circ}, 22^{\circ}, 31^{\circ}, 39^{\circ}, 45^{\circ}, 50^{\circ}\right)$.

The arrival direction of a muon is determined for each triggered PRC in the lower layer and binned into 13 directions based on the specific location of the PRC triggered in the upper layer from among the 13 PRCs, one directly above (central PRC) and six each on either side of the central PRC, as also shown in Figure 4. Corresponding projection angles are $0^{\circ}, 11^{\circ}, 22^{\circ}, 31^{\circ}, 39^{\circ}, 45^{\circ}$, and $50^{\circ}$. This directional binning has been carried out in each of the two orthogonal projection planes, thereby generating a map of $13 \times 13=169$ solid-angle directions of the muons, as shown in Figure 5. The contents of the 169 directions have been recorded once every $10 \mathrm{~s}$, thereby generating a continuous record of the directional flux of muons in the sky.

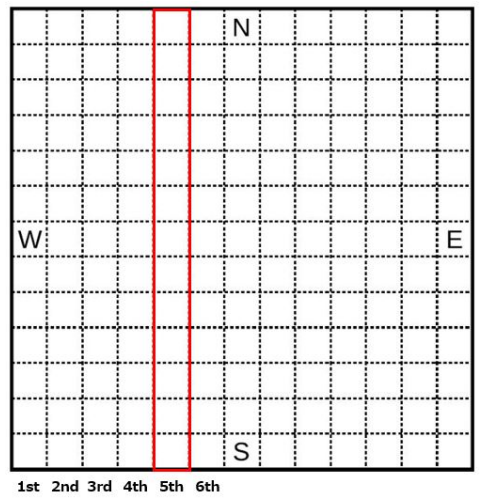

Figure 5: The field of view of the muon telescope (169 directions). The data from 13 directions indicated by the red rectangle were used to make a 2D map shown in Figure 6. 


\section{The solar time anisotropy}

Using the GRAPES-3 large area and high-resolution muon telescope explained in the previous section, we can obtain two kinds of 2D map of the distribution of the GCR intensity. One is a "snapshot" of the sky, and another is a "scanned image" of that.

In the "snapshot" case, all the contents of the 196 directions recorded simultaneously in some time interval make one 2D map of the distribution of the GCR intensity. This visualization is a sky photo taken by a muon camera of $169(13 \times 13)$ pixels. Beautiful applications of this $2 \mathrm{D}$ visualization method have already been published elsewhere $[7,8,11]$.

In the "scanned image" case, the field of view of the muon telescope is divided into 13 northsouth lines parallel to the meridian. To make one 2D map of the distribution of the GCR intensity, only one north-south line between them is used. This visualization is a sky photo scanned by a muon line sensor of 13 in-line pixels. The horizontal axis of the obtained map is the solar time, as same as Figure 1. In this case, 13 maps are obtained for one duration of the observation. This visualization method is our present proposal.

Here, in Figure 6, we will show an example of a 2D map of the distribution of the GCR intensity viewed by the north-south line of the 5th counted from the west (indicated by a red rectangle in Figure 5). The data used to obtain this map had been accumulated during 14 years from 2004 to 2017. So all the transient variations are expected to have been smeared out and this map shows the steady-state solar time anisotropy. From this map, we can easily see the single broad peak is there at about $13 \mathrm{~h}$ solar time and $0^{\circ}$ latitude. This result is the same as the result obtained before by the time series analysis using the GRAPES-3 muon telescope [3,4]. The result is not inconsistent with the result obtained by taking into the drift effect [6,20]. All 2D maps viewed by other 12 lines show similar images.

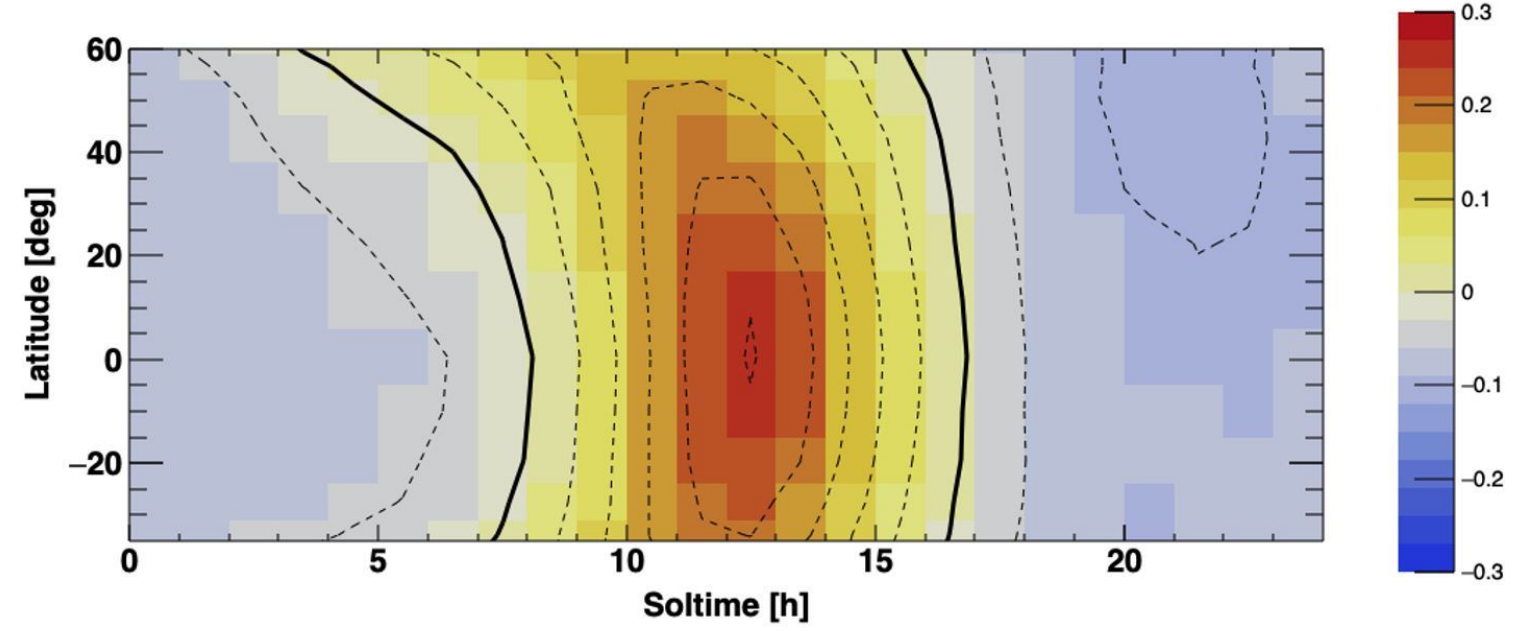

Figure 6: An example of a 2D map of the distribution of the GCR intensity viewed by the north-south line of the 5th counted from the west (indicated by a red rectangle in Figure 5). 


\section{Data processing procedures}

The following procedures were employed to get the 2D map of the GCR intensity for 14 years of data presented in the previous section.

In the first stage, we got the hourly values of the muon intensity deviation from the average by the following procedures.

1. The 4 minutes counting rate of each direction was adopted as a basic unit. For the 2D map shown in Figure 6, 14 years of data set (from 2004 to 2017) are used.

2. The average muon counting rate for each calendar year were calculated.

3. To remove the geometrical differences, such as the solid angle or the zenith angle dependence for each direction, and to get the relative value, the deviation from the average for each 4 minutes counting rate was calculated. From now on, all counting rate deviations are expressed as the percent values. After this process, one hour counting rate deviation was calculated by taking the average for each hour.

4. The barometric correction was done to get the proper muon intensity deviation. For this correction, barometric correction coefficient for muon intensity deviation at Ooty had been separately obtained.

At this point, we had the hourly values of the muon intensity deviation for every 169 directions during 14 years. Since we were interested in the steady-state solar time anisotropy, we applied a 24 hours high-pass filter along with the following procedures, as the second stage.

1. The running average of 24 hours was calculated for all the hourly data of each direction.

2. The difference between the hourly value and the running average was taken for each corresponding hour.

3. For every 169 directions, each hourly data was superimposed to make the one solar day (24 hours) data.

At this point, we got 24 hours data for every 169 directions. Our "scanned image" visualization method can construct one 2D map using the processed data sets corresponding to one of the northsouth lines parallel to the meridian. In the final stage we made the $132 \mathrm{D}$ maps by the following procedures.

1. One 2D map was created by arranging the data set corresponding to some north-south line in parallel from left $(0 \sim 1 \mathrm{~h})$ to right $(23 \sim 24 \mathrm{~h})$.

2. Since the obtained 2D map represented a single scan of the sky around the Earth, the horizontal axis of the map was shifted by the east-west tilt of the corresponding direction. So it was necessary to correct the time corresponding to the angle. Finally, the horizontal axis of all the maps obtained were aligned with the solar time coordinate with the direction of the sun at $12 \mathrm{~h}$.

Our proposed scheme generates 13 2D maps of the distribution of the GCR intensity. 


\section{Summary}

In this final section, we will summarize this work presented here and discuss some points on the result.

1. To know the directional distribution of GCR helps to investigate the aspect of the IMF around the Earth.

2. Since the GRAPES-3 muon telescope can provide a high statistics, directional measurement of the muons, we can draw high-resolution 2D maps of the distribution of the GCR intensity.

3. So we will get much information about the intensity of GCR on the sky compared to the traditional ways of analyses.

4. We propose here two methods for visualization using our muon telescope.

5. One method has been applied previously to be useful for transient phenomena $[7,8,11]$.

6. Another method is demonstrated here to show the steady-state solar time anisotropy of GCR. And the result is consistent with the previous works done by the traditional way of analysis.

We conclude that the 2D visualization method for the solar time anisotropy of GCR below 1 $\mathrm{TeV}$ has been developed with the above summary. Expectedly, this method replicates a 2D structure guessed by the traditional method [3, 4].

In the present study, a long-term stable distribution is presented to show how effective this method is. In the future, we would like to make various necessary corrections and create 2D maps by handling data for a shorter period to confirm the phenomena that have been revealed so far and apply the method developed here to clarify the mechanisms behind new phenomena.

\section{References}

[1] T. Nonaka et al., Phys. Rev. D 74 (2006) 52003.

[2] P. Subramanian et al., Astron. Astrophys. 494 (2009) 1107.

[3] P.K. Mohanty et al., Pramana J. Phys. 81 (2013) 343.

[4] H. Kojima et al., Astroparticle Phys. 62 (2015) 21.

[5] P.K. Mohanty et al., Astroparticle Phys. 79 (2016) 23.

[6] R. Modzelewska et al., Solar Phys. 294 (2019) 148.

[7] T. Nonaka, Ph. D. thesis, Osaka City University (2005).

[8] T. Nonaka et al., Proc. 29th ICRC, Pune, 1 (2005) 359.

[9] P.K. Mohanty et al., Phys. Rev. Lett., 117 (2016) 171101.

[10] P.K. Mohanty et al., Phys. Rev. D97 (2018) 082001. 
[11] B. Hariharan et al., Phys. Rev. Lett., 122 (2019) 105101.

[12] S.K. Gupta et al., Nucl. Instrum. Methods A 540 (2005) 311.

[13] Y. Hayashi et al., Nucl. Instrum. Methods A 545 (2005) 643.

[14] S.K. Gupta et al., Phys. Rev. D68 (2003) 052005.

[15] S.C. Tonwar et al., Int. J. Mod. Phys. A 20 (2005) 6852.

[16] S.K. Gupta et al., Nucl. Phys. B Proc. Suppl. 196 (2009) 153.

[17] H. Tanaka et al., J. Phys. G: Nucl. Part. Phys. 39 (2012) 025201.

[18] H. Kojima et al., Phys. Rev. D91 (2015) 121303(R).

[19] H. Kojima et al., Phys. Rev. D98 (2018) 022004.

[20] J. Kota and J.R. Jokipii, Astrophys. J. 265 (1983) 573. 